\title{
Evaluation the effect of preserving intercostobrachial nerve in axillary dissection for breast cancer patient
}

\author{
Prakasit Chirappapha, Methas Arunnart, Panuwat Lertsithichai, Chairat Supsamutchai, Thongchai \\ Sukarayothin, Monchai Leesombatpaiboon
}

Department of Surgery, Faculty of Medicine Ramathibodi Hospital, Mahidol University, Bangkok, Thailand

Contributions: (I) Conception and design: All Authors; (II) Administrative support: None; (III) Provision of study materials or patients: All Authors; (IV) Collection and assembly of data: All Authors; (V) Data analysis and interpretation: All Authors; (VI) Manuscript writing: All authors; (VII) Final approval of manuscript: All authors.

Correspondence to: Dr. Chairat Supsamutchai, MD. Department of surgery, Ramathibodi Hospital, Mahidol University, Bangkok 10400, Thailand. Email: pogeneral2007@hotmail.com.

Background: The Intercostobrachial nerve (ICBN) is responsible for sensory function in the axillar and upper arm. The majority of surgeons routinely sacrifice the ICBN during axillary lymph node dissection (ALND) because of technical difficulties. Therefore, the aim of this study was to assess the effects of the preservation or division of the ICBN on the incidence of post-operative sensory disturbance, health-related quality of life (HRQOL), and the physical functions of the upper limbs.

Methods: We performed a randomized double-blind trial comparing the incidence of sensory disturbance, HRQOL and physical functions of upper limbs in the preservation and the removal of the ICBN. Clinicians performed sensory evaluation at 2 weeks and 3 months after surgery. The sensory evaluation included questionnaires (subjective evaluation) and physical examination (objective evaluation) to evaluate sensory disturbance of the upper arm. HRQOL and physical function of upper limbs was accessed before surgery and at three months after surgery, using Short Form-36 and QuickDASH questionnaires, both in Thai language versions.

Results: At the end of the surgical procedures there were 15 patients in the preserved group (group P) and 28 patients in the non-preserved group (group N). In as-treated analysis, there was no significant difference between the groups in pain, sensory loss, physical examination of touch and pinprick sensation, and areas of sensory dullness. HRQOL found that the reported pain in $\mathrm{P}$ group was higher than $\mathrm{N}$ group in both intention-to-treat and as-treated analysis. In the QuickDASH scores of physical functions of the upper limbs there was a significant difference, 9.1 in group $\mathrm{P}$ and 20.5 in group $\mathrm{N}(\mathrm{P}=0.013)$.

Conclusions: ICBN preservation provides no benefit to improving sensation, but there are benefits in HRQOL and physical functions of upper limbs at three months after surgery.

Keywords: Intercostobrachial nerve preservation; axillary lymph node dissection; breast cancer

Submitted Jul 16, 2019. Accepted for publication Sep 27, 2019.

doi: 10.21037 /gs.2019.10.06

View this article at: http://dx.doi.org/10.21037/gs.2019.10.06

\section{Introduction}

Axillary lymph node dissection (ALND) is the standard treatment for staging and local management in most of node-positive breast cancer patients (1-3). But ALND may result in arm lymphedema, sensory disturbance, and shoulder dysfunction, which compromise functionality and health-related quality of life (HRQOL) (4). The intercostobrachial nerve (ICBN) injury during ALND is believed to be responsible for the development of sensory disturbance in both persistent pain and loss of sensory function in the region supplied (5-8). It has been 


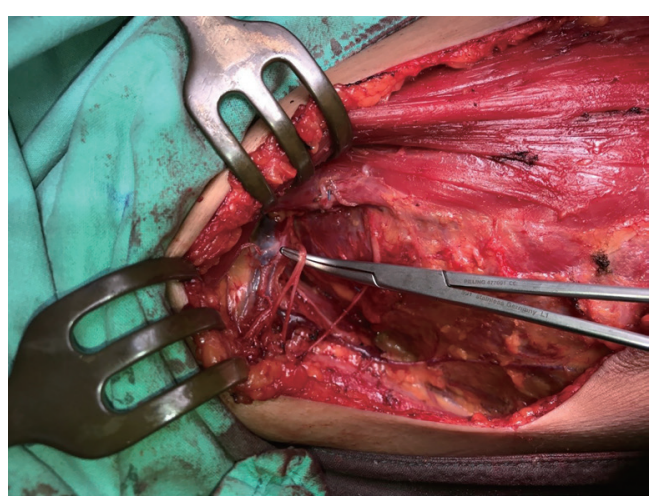

Figure 1 The intercostobrachial nerve.

shown that preservation of the ICBN decreases the risk of sensory disturbance, but the evidence was limited to a few randomized controlled trials (RCTs) with the variable quality of the studies (9-11). Therefore, the aim of this study was to assess the effect of preservation or division of the ICBN on the incidence of post-operative sensory disturbance, HRQOL and physical function of upper limb.

\section{Methods}

\section{Study design}

We performed a randomized double-blind trial comparing the incidence of sensory disturbance, HRQOL and physical function of upper limb in the preservation of the ICBN with the division of the ICBN. The study was registered on Thai Clinical Trials Registry (TCTR) number TCTR20180908002, and approved by the Office of The Committee for Research, Faculty of Medicine Ramathibodi Hospital Mahidol University (Protocol number ID 04-59-08).

\section{Participants}

Women who were aged 16 years or older who had ALND for primary breast cancer at Ramathibodi Hospital from September 2016 to September 2017 were recruited. Patients were not eligible if they planned to have breast reconstruction surgery, previous axillary surgery, preoperative difficulties of arm movement or neuropathy at upper limb, could not identified ICBN intraoperative or tumor was invaded ICBN, and inability to consent to the study. All patients provided written informed consent before surgery.

\section{Interventions}

The surgeries were performed by 6 surgeons, using the same technique. ALND was begun with identified lateral margin of Pectoralis major muscle and dissected to interpectoral space until the axillary vein was reached. ICBN was identified where in emerges into the thoracic wall at second intercostal space. There were ICBN branches on the peripheral side in many cases. When a branch distributing to the upper arm was preserved, this was classified as successful ICBN preservation (Figure 1).

\section{Outcomes}

Clinicians performed sensory evaluation at 2 weeks and 3 months after surgery. The sensory evaluation included questionnaires (subjective evaluation) and physical examination (objective evaluation) to evaluate sensory disturbance of the upper arm. Subjective sensory evaluation included pain score and degree of sensory loss at upper arm. Pain score (PS) at upper arm was measured using visual analog scale (VAS) and reported as no pain if PS $=0$, mild pain if PS $=1-3$, moderate to severe pain if PS $\geq 4$. In patients who decreased sensation at upper arm, degree of sensory loss at upper arm was measured using VAS. Anesthesia was defined as degree of sensory loss score $=10$ and degree of sensory loss score $=0$, if no change of sensation.

Objective sensory evaluation included physical examination that focused on touch sensation, pinprick sensation and area of sensory dullness at upper arm. Touch sensation was examined by cotton ball-light touch at $1 \mathrm{~cm}$ below axillary hairline. Pinprick sensation was examined using $10 \mathrm{~g}$ monofilament at $1 \mathrm{~cm}$ below axillary hairline. The degree of touch and pinprick sensation compared to the contralateral arm was classified as no difference, decrease sensation and increase sensation. The area of sensory dullness was tested by cotton ball-light touch and monofilament, started from olecranon process of the ulna to axillary hairline then identified the point that firstly decreased sensation. The area of sensory dullness was calculated as ratio $\mathrm{A} / \mathrm{B}$. A was the distance from axillary hairline to point that firstly decreased sensation and $\mathrm{B}$ was the distance from axillary hair line to olecranon process of ulna (Figure 2) HRQOL was accessed before surgery and at 3 months after surgery using Short form-36 questionnaires (SF-36). SF-36 consists of 8 domains: 


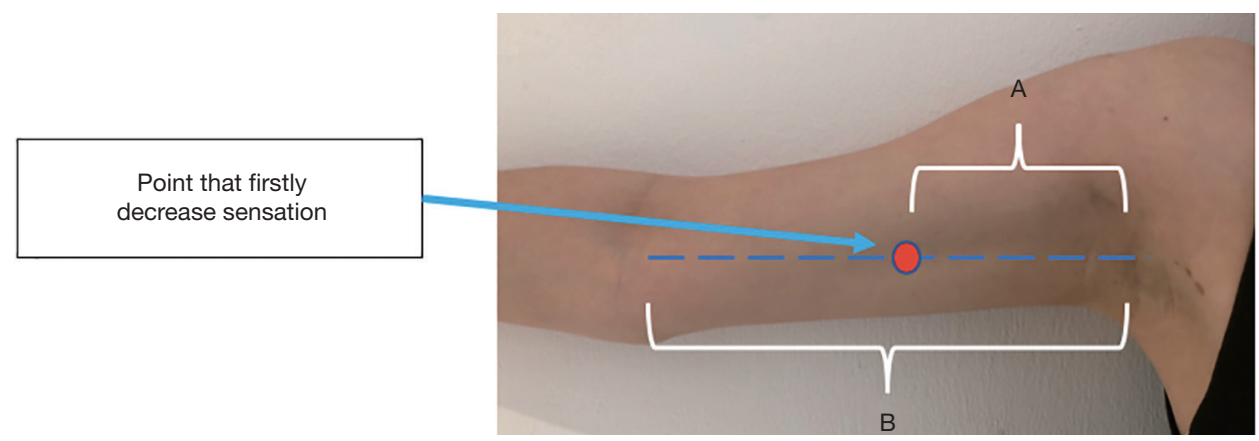

Figure 2 Area of sensory dullness examination. A was the distance from axillary hairline to point that firstly decreased sensation. B was the distance from axillary hair line to olecranon process of ulna.

physical function, role physical function, role emotional function, vitality, mental health, social function, bodily pain, and general health. The raw scores of each subscale were converted to a range from 0 to 100 . Higher scores indicated better HRQOL. The reliability and validity of the Thai version of SF-36 has been verified $(12,13)$. Results are calculated as described by: http://www.rand.org/health/ surveys_tools/mos/mos_core_36item_scoring.html.

Physical function of upper limb was assessed before surgery and at 3 months after surgery using QuickDASH questionnaires. QuickDASH consists of 11 items to measure physical function and symptoms (14). At least 10 of the 11 items must be completed for a score to be calculated. A higher score indicates greater disability. The Thai version of QuickDASH has been verified (15).

All of pre-operative data was collected at ward before surgery. At 3 months after surgery, data was collected mainly at outpatient clinic. If patients did not cooperate to answer questionnaire at outpatient clinic, data will be collected by telephone.

\section{Sample size}

\section{Randomisation and blinding}

Patients were randomly assigned intraoperative to preserve or divide the ICBN only when the ICBN was intact. To randomly assign a patient, research staff at the operating room telephoned primary investigator to obtain the treatment allocation in the seal envelopes. Treatment allocation was blinded from patients and clinicians who analysing the data.

\section{Statistical methods}

The association between ICBN status and pain, decrease sensation, touch and pinprick sensations were analyzed by chi-square test. The association between ICBN status and degree of sensory loss, area of sensory dullness, HRQOL, and physical function of upper limb outcome (SF-36 and QuickDASH score) were analyzed by Mann-Whitney $U$ test. Differences were considered to be significant at $\mathrm{P}$ value less than 0.05 . Data analyses were done according to the Intention-to-treat and as-treated analysis. Statistical analysis was performed using STATA version14.1.

\section{Results}

\section{Baseline characteristics of the participants}

A total of 157 patients underwent baseline assessment and 43 patients were eligible (Figure 3). Patients were randomly assigned to either the ICBN-preserved group (group P) for 22 patients, or non ICBN-preserved group (group N) for 21 patients. There were 7 patients in group P (31\%) who failure to preserved ICBN after randomization. This means that 15 patients were in actually preserved group (group AP) and 28 patients were in actually non-preserved group (group $\mathrm{AN}$ ) in as-treated analysis. Both groups were comparable with regard to clinical background characteristics (Table 1).

The mean age of all the subjects was 56.6 years old. Most surgical procedure was mastectomy. Median number of removed lymph nodes was 16 (range, 13-24). Neoadjuvant chemotherapy was administered in 16 patients (37\%) and postoperative adjuvant chemotherapy in 25 patients $(58 \%)$. Adjuvant trastuzumab was administered in 7 patients (17\%). Postoperative hormone therapy was administered in 30 patients $(70 \%)$ and 27 patients $(63 \%)$ received postoperative irradiation. During the follow-up period, no patient died and no local recurrence. Distant metastases were found in 3 cases.

Number of patients who responded to sensory evaluation 


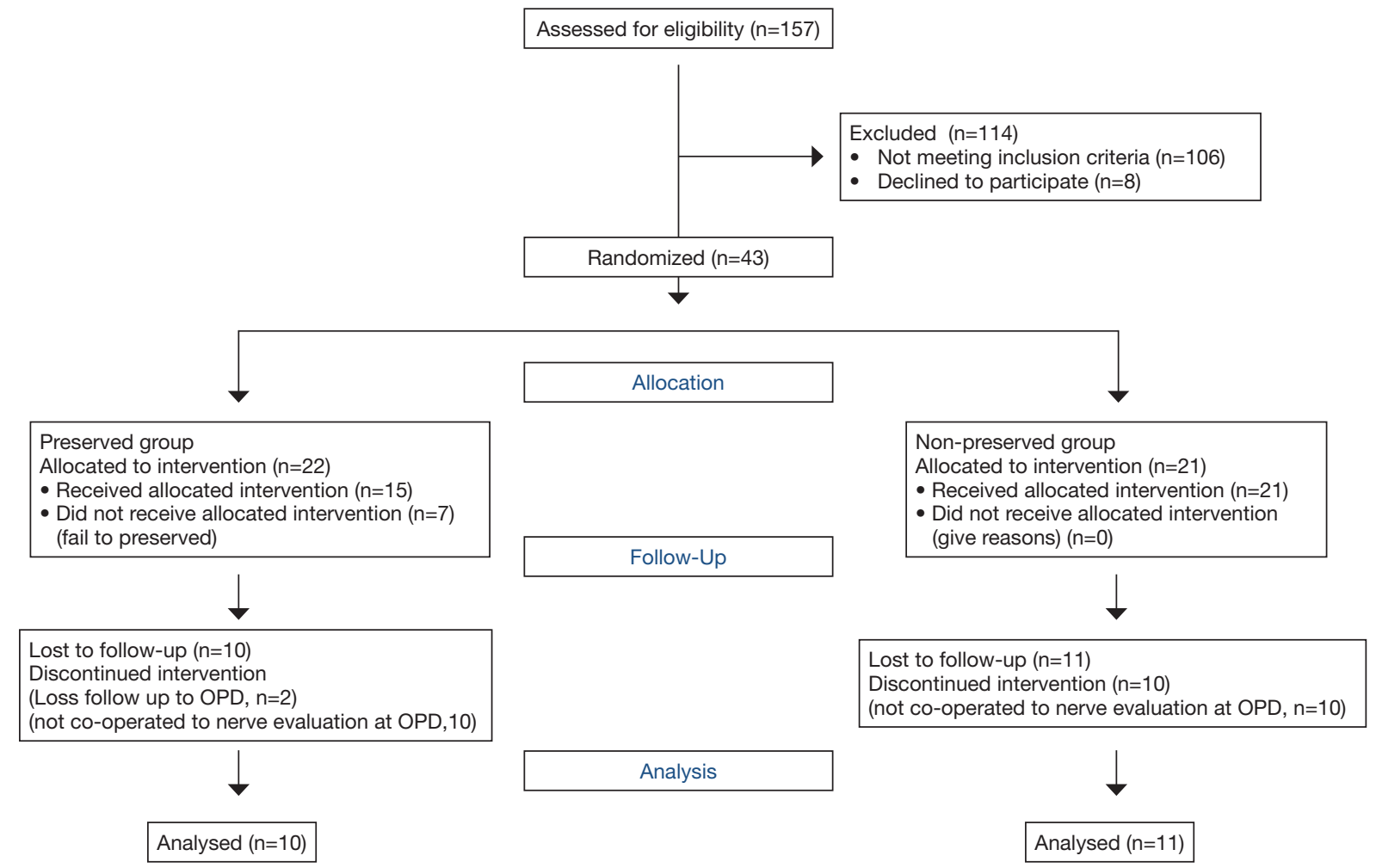

Figure 3 Trial profile.

at 2 weeks and 3 months after surgery were $38(88 \%)$ and 21 (49\%), respectively. No significant difference of patient characteristic in patients who response to sensory evaluation in both intention-to-treat and as-treated analysis.

Number of patients who responded to HRQOL and physical function of upper limb evaluation at preoperative and 3 months after surgery were $43(100 \%)$ and $36(84 \%)$, respectively. In 36 patients who responded to HRQOL and physical function of upper limb evaluation at 3 months after surgery, no significant difference of patient characteristic between both groups except adjuvant chemotherapy received. Patients in group $\mathrm{P}$ received adjuvant chemotherapy more than group $\mathrm{N}$ (Table 2).

\section{Sensory evaluation}

In intention to treat analysis, the percentage of patients who had pain at upper arm at 2 weeks was $26 \%$ in group $\mathrm{P}$ and $53 \%$ in group $\mathrm{N}$, which decreased with time in both groups. At 3 months, none of the patients in group $\mathrm{P}$ had pain whereas $27 \%$ in group $\mathrm{N}$ had pain at upper arm. The different of percentage of patients who had pain did not differ significantly between the groups at any time point (Table 3).

The rate of sensory loss at 2 weeks and 3 months were $79 \%, 55 \%$ in group $\mathrm{P}$ and $79 \%, 70 \%$ in group $\mathrm{N}$. The degree of sensory loss at 2 weeks and 3 months were 3 and 1 , respectively in both groups. No significant difference in percentage of patients with sensory loss and degree of sensory loss at upper arm between both groups in any time point.

The rates of touch sensation at 2 weeks after surgery was $26 \%$ no difference, $74 \%$ decrease sensation and no patient with increase sensation in group $\mathrm{P}$. In group $\mathrm{N}$ the rates were $21 \%, 74 \%$ and $5 \%$, respectively. At 3 months after surgery, the rate of no difference was $70 \%$ and decrease sensation was $30 \%$ in group P. In group N, the rate of no difference was $64 \%$ and decrease sensation was $36 \%$. No patient in both groups had increase sensation. No significant difference in touch sensation between both groups in any time point.

The result of pinprick sensation at 2 weeks after surgery, rates of no difference, decrease sensation and increase sensation was $32 \%, 63 \%$ and $5 \%$ in group $\mathrm{P}$ and $16 \%$, 
Table 1 Patient characteristic

\begin{tabular}{|c|c|c|c|c|c|c|c|}
\hline Characteristic & $\begin{array}{c}\text { All case } \\
(n=43)\end{array}$ & \multicolumn{3}{|c|}{ Intention to treat analysis } & \multicolumn{3}{|c|}{ As treated analysis } \\
\hline Age (years), mean \pm SD & $56.6 \pm 10.1$ & $58.6 \pm 10.7$ & $55.10 \pm 9.2$ & 0.259 & $58.9 \pm 9.8$ & $55.8 \pm 11.1$ & 0.333 \\
\hline Clinical staging, n [\%] & & & & 0.449 & & & 0.256 \\
\hline TO & $1[2]$ & $1[5]$ & $0[0]$ & & $1[7]$ & $0[0]$ & \\
\hline $\mathrm{T} 2$ & $22[51]$ & 13 [59] & 9 [43] & & $10[67]$ & 12 [43] & \\
\hline T3 & $8[19]$ & $3[14]$ & $5[24]$ & & $1[7]$ & 7 [25] & \\
\hline $\mathrm{T} 4$ & 5 [12] & $3[14]$ & $2[10]$ & & $1[7]$ & $4[14]$ & \\
\hline $\mathrm{N}$ stage, $\mathrm{n}[\%]$ & & & & 0.203 & & & 0.897 \\
\hline N2 & $2[5]$ & 2 [9] & $0[0]$ & & $1[7]$ & $1[4]$ & \\
\hline \multicolumn{8}{|l|}{ Evaluation of LN, median } \\
\hline No. of positive LN & $2[1-5]$ & $2[1-5]$ & $2[1-3]$ & 0.554 & $2[1-9]$ & $2[1-4]$ & 0.485 \\
\hline No. of removed LN & 16 [13-24] & 17 [14-22] & 16 [13-25] & 0.932 & $15.5[12.5-24.5]$ & $19[15-24]$ & 0.395 \\
\hline Surgical procedure, $\mathrm{n}$ [\%] & & & & 0.300 & & & 0.459 \\
\hline Mastectomy & 42 [98] & $22[100]$ & 20 [95] & & 15 [100] & 27 [96] & \\
\hline Breast conservative surgery & $1[2]$ & $0[0]$ & $1[5]$ & & $0[0]$ & $1[4]$ & \\
\hline Neoadjuvant chemotherapy, n [\%] & 16 [37] & $9[41]$ & 7 [33] & 0.607 & $6[40]$ & $10[36]$ & 0.782 \\
\hline
\end{tabular}

$74 \%$ and $10 \%$ in group N, respectively. At 3 months after surgery, rates of no difference and decrease sensation were $60 \%$ and $40 \%$ in group $\mathrm{P}$ and $36 \%$ and $64 \%$ in group $\mathrm{N}$, respectively. No patient in both groups had increase sensation. No significant difference in pinprick sensation between both groups in any time point.

The areas of touch sensory dullness at 2 weeks and 3 months were $0.2,0$ in group $\mathrm{P}$ and $0.3,0$ in group $\mathrm{N}$, respectively. The areas of pinprick sensory dullness at 2 weeks and 3 months were $0.3,0$ in group $\mathrm{P}$ and $0.2,0.2$ in group N, respectively. No significant differences in the area of sensory dullness of touch and pinprick sensation between both groups at any time point.

In as-treated analysis, no significant in pain, sensory loss, physical examination of touch and pinprick sensation, and area of sensory dullness (Table 4).

\section{HRQOL and physical function of upper limb}

No significant difference in pre-operative SF-36 and QuickDASH score between both groups (Table 5).

At 3 months after surgery, no significant difference of SF-36 domains except bodily pain. Bodily pain domain in ICBN-preserved group was higher than non ICBNpreserved group in both intention-to-treat and as-treated analysis (Table 6).

QuickDASH scores at 3 months after surgery was 9.1 in group $\mathrm{P}$ and 20.5 in group $\mathrm{N}(\mathrm{P}=0.013)$. QuickDASH score 
Table 2 Patient characteristic of 36 patients who were responders for HRQOL and physical function of upper arm evaluation at 3 months after surgery

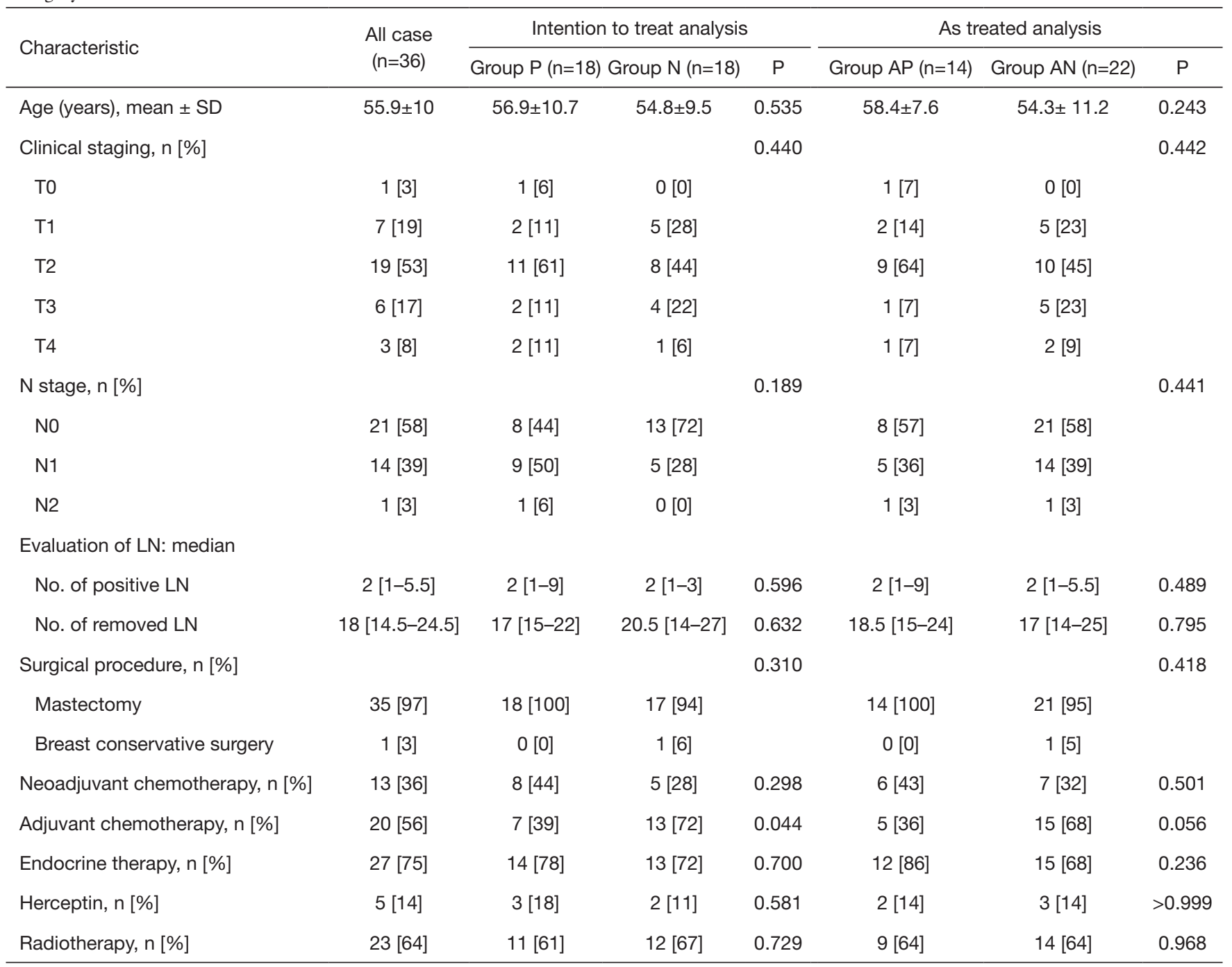

in ICBN-preserved group was higher than non ICBNpreserved group, but the difference was significant only in intention-to-treat analysis.

\section{Discussion}

Now a day there are many ongoing researches in clinically node-negative and sentinel lymph node positive breast cancer for decrease unnecessary ALND (1-4). But in clinically node-positive breast cancer ALND still standard treatment. Patient who had ALND may result in lymphedema, nerve injury, and shoulder dysfunction, which compromise functionally and HRQOL (1-9). ICBN is believed to be responsible for sensory function in the axillar and upper arm. The majority of surgeons routinely sacrifice the ICBN because of technical difficulty. ACOSOG Z0011 (5) and IBCSG23-01 (3) were show sensory disturbance 1 year after ALND 39\% and 18\% respectively

In 1998, Abdullah et al. (9) reported the first randomized controlled trial that shown the benefit of ICBN preservation. Preservation was found to reduce the incidence of sensory deficit at 3 months after surgery but no detail of neurological assessment in this study.

In 2003, Torresan et al. (11) reported randomized controlled trial, in which the pain sensitivity of the arm at 
Table 3 Sensory evaluation in patient with preservation (group P) and non-preservation (group N)

\begin{tabular}{|c|c|c|c|c|c|c|}
\hline \multirow{2}{*}{ Sensory evaluation } & \multicolumn{6}{|c|}{ Intention to treat analysis } \\
\hline & Group P ( $n=19)$ & Group N (n=19) & $\mathrm{P}$ & Group P $(n=10)$ & Group N (n=11) & $\mathrm{P}$ \\
\hline Pain, n [\%] & & & 0.145 & & & 0.204 \\
\hline No pain (PS =0) & $14[74]$ & $9[47]$ & & 10 [100] & 8 [73] & \\
\hline Moderate to Severe pain ( $P S \geq 4$ ) & $3[16]$ & $3[16]$ & & $0[0]$ & $2[18]$ & \\
\hline Decrease sensation, n [\%] & 15 [79] & 15 [79] & $>0.999$ & $6[55]$ & $7[70]$ & 0.466 \\
\hline Degree of sensory loss & $3[1-5]$ & $3[1-5]$ & 0.754 & $1[0-3]$ & $1[0-5]$ & 0.826 \\
\hline Touch sensation, n [\%] & & & 0.574 & & & 0.757 \\
\hline Increase sensation & $0[0]$ & $1[5]$ & & $0[0]$ & $0[0]$ & \\
\hline Pinprick sensation, n [\%] & & & 0.475 & & & 0.279 \\
\hline No difference & 6 [32] & $3[16]$ & & $6[60]$ & $4[36]$ & \\
\hline Decrease sensation & $12[63]$ & $14[74]$ & & $4[40]$ & $7[64]$ & \\
\hline Increase sensation & $1[5]$ & $2[10]$ & & $0[0]$ & $0[0]$ & \\
\hline \multicolumn{7}{|l|}{ Area of sensory dullness: median } \\
\hline Touch sensation & $0.2[0-0.4]$ & $0.3[0.1-0.5]$ & 0.448 & $0[0-0.25]$ & $0[0-0.35]$ & 0.936 \\
\hline Pinprick sensation & $0.3[0-0.4]$ & $0.2[0.1-0.4]$ & 0.593 & $0[0-0.2]$ & $0.2[0-0.3]$ & 0.223 \\
\hline
\end{tabular}

2 days, 40 days and 3 months after surgery. Pain sensitivity of arm was evaluated subjectively and objectively. There were more asymptomatic patients and more patients with normal neurological examinations in the ICBN preservation group than non-preserved group. No detail of demographic data in both groups. In 2014, Taira et al. (16) reported multicenter longitudinal case-controlled study measuring sensory change and HRQOL at 1, 6, 12, 24 months after surgery. Sensory change was evaluated subjectively and objectively. HRQOL was assessed using multiple regression analysis of FACT-G, Breast cancer subscale, FACT-B TOI score. The results shown the benefit of ICBN preservation in reduction area with long-term axillary hypoesthesia, but has no influence on improvement of pain and HRQOL and adjuvant chemotherapy markedly decreased HRQOL of patients.

In this study, sensory disturbance was evaluated subjectively and objectively. We found that ICBN preservation had no significantly benefit in subjective sensory evaluation at 3 months after surgery. Only few patients had moderate to severe pain at 3 months after operation $(0 \%$ in preserved group and $18 \%$ in nonpreserved group). Fifty-five percent in preserved group and $70 \%$ in non-preserved group and decrease sensation at upper arm at 3 months after surgery but low degree of sensory loss.

There was no significant difference in touch or pinprick sensation between patients with preserved and nonpreserved ICBN. The results of intention-to-treat analysis is as same as to as-treated analysis. The area of sensory dullness was the objective evaluation that tried to estimate the degree of sensory loss measured by light touch and pinprick test that expressed in ratio of distance of sensory dullness area and distance of upper arm (axillary hair line to olecranon process). In this study, no significant difference in the area of sensory dullness. There are many questionnaires for HRQOL evaluation. In this study, we want questionnaires that world-wide used, Thai version have been 
Table 4 Sensory evaluation in patient with actually preservation and actually non-preservation

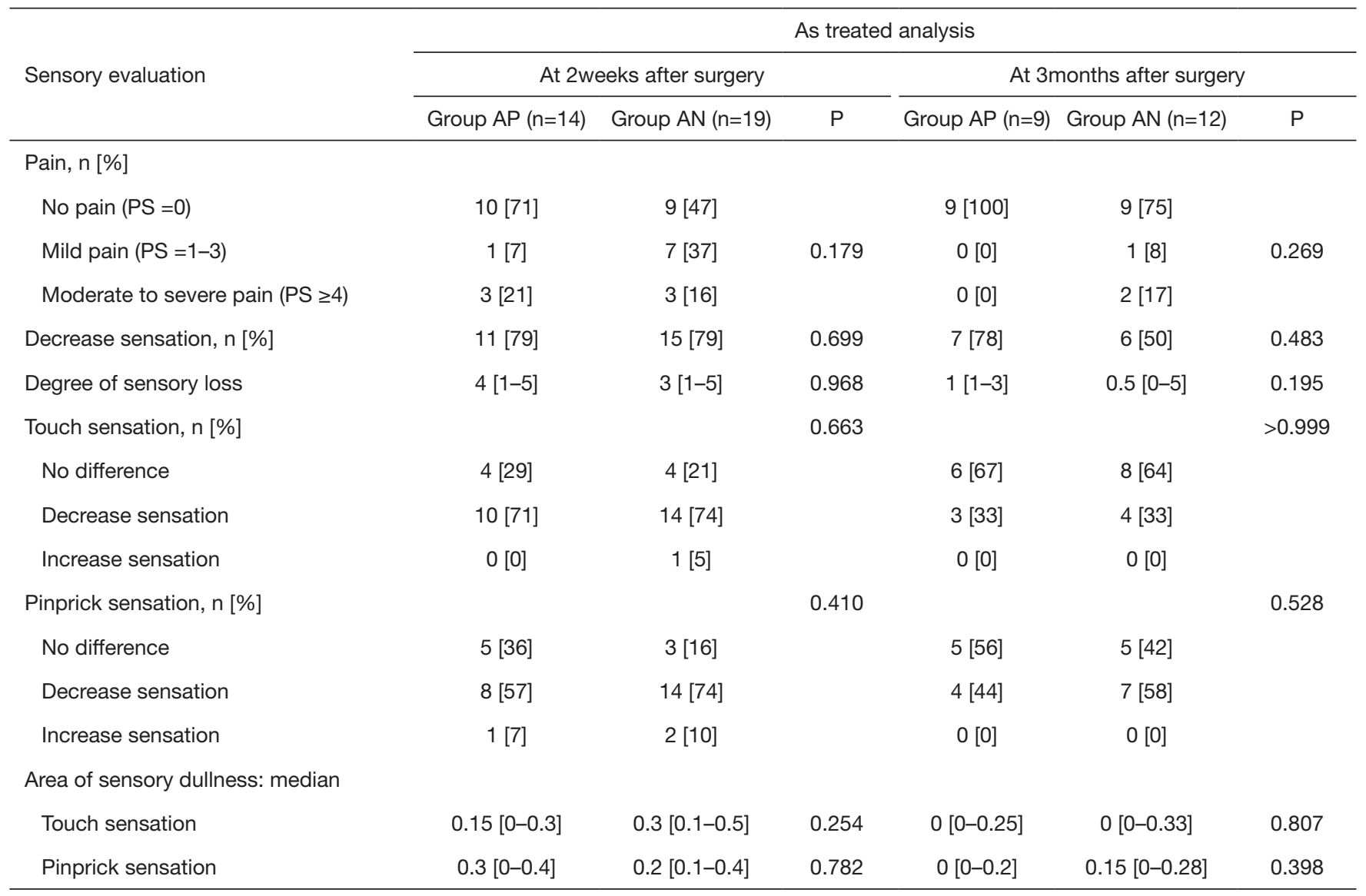

Table 5 HROQL and physical function of upper arm at preoperative

\begin{tabular}{|c|c|c|c|c|c|c|}
\hline Outcome & \multicolumn{3}{|c|}{ Intention-to-treat analysis } & \multicolumn{3}{|c|}{ As-treated analysis } \\
\hline \multicolumn{7}{|l|}{ SF-36: median } \\
\hline Physical function & $87.5[60-100]$ & 80 [60-90] & 0.244 & $87.5[60-100]$ & 80 [60-95] & 0.432 \\
\hline Role physical function & $62.5[0-100]$ & $50[0-100]$ & 0.624 & $70[0-100]$ & $50[0-100]$ & 0.592 \\
\hline Vitality & $55[50-75]$ & $65[50-70]$ & 0.911 & $55[50-80]$ & $65[50-70]$ & 0.974 \\
\hline Mental health & $68[60-80]$ & 66 [52-68] & 0.197 & $68[60-80]$ & $66[52-72]$ & 0.260 \\
\hline Social function & $87.5[50-100]$ & 75 [75-87.5] & 0.923 & $93.75[50-100]$ & 77.5 [75-87.5] & 0.727 \\
\hline Bodily pain & $100[77.5-100]$ & $90[67.5-100]$ & 0.393 & 93.75 [77.5-100] & $90[77.5-100]$ & 0.945 \\
\hline
\end{tabular}


Table $6 \mathrm{HROQL}$ and physical function of upper arm at 3 months after surgery

\begin{tabular}{|c|c|c|c|c|c|c|}
\hline Outcome & \multicolumn{3}{|c|}{ Intention-to-treat analysis } & \multicolumn{3}{|c|}{ As-treated analysis } \\
\hline \multicolumn{7}{|l|}{ SF-36: median } \\
\hline Physical function & 85 [60-95] & 77.5 [60-85] & 0.127 & 85 [60-95] & 80 [60-85] & 0.247 \\
\hline Role physical function & $100[50-100]$ & $62.5[0-100]$ & 0.105 & $100[50-100]$ & $75[0-100]$ & 0.189 \\
\hline Vitality & $67.5[55-75]$ & $62.5[50-65]$ & 0.355 & $70[60-80]$ & $60[50-65]$ & 0.160 \\
\hline Mental health & 70 [68-80] & $68[60-76]$ & 0.414 & 70 [68-80] & $68[60-76]$ & 0.411 \\
\hline Social function & $100[75-100]$ & $87.5[75-100]$ & 0.373 & $100[75-100]$ & $87.5[75-100]$ & 0.513 \\
\hline Bodily pain & $100[90-100]$ & 77.5 [55-90] & $0.007^{\star}$ & $100[90-100]$ & $77.5[57.5-90]$ & $0.009^{*}$ \\
\hline
\end{tabular}

verified and represented physical function and symptoms of upper limb, so we selected SF-36 questionnaires that have 8 domains including physical function, role physical function and bodily pain. The physical function of upper limb was included in the outcome of this study due to we had a hypothesis that there were few questions in the HRQOL questionnaires that represented the physical function of upper limb.

HRQOL questionnaires maybe underestimate the problem about physical function of upper limb. We selected QuickDASH questionnaires due to Thai version have been verified and had just 11 items. The patients were comfortable to answer the questionnaires after answered 36 items of SF-36. No significant difference of all 8 domains of SF-36 except bodily pain domain at 3 months after surgery in both intention-to-treat and as-treated analysis. Bodily pain domain in preserved group was significantly higher than non-preserved group that mean at 3 months after surgery, patients who ICBN-preserved had better HRQOL.

At 3 months after surgery, QuickDASH score of ICBN preserved group was significantly lower than non-preserved group (higher score indicates greater disability). But significant difference only in intention-to-treat analysis. We concluded that ICBN-preserved can improved physical function of upper arm at 3 months after surgery.

The limitation in this study was small sample size and there is confounding factor that causing difference in HRQOL and physical function of upper arm in this study was patients in non-preserved group received adjuvant chemotherapy more than preserved group. Tingling at hand is the common problem in the patients who received chemotherapy but it is not associate to complication after ICBN resection. SF-36 and QuickDASH has questionnaires about pain at upper limb but not separate the symptoms of upper arm and hand. This factor may cause lower bodily pain domain in SF-36 and higher QuickDASH score and overestimated the benefit of ICBN preservation. Ideally, a longer-term follow up would enable us to better understand the outcomes of this type of surgery.

In conclusion, this randomized controlled trial demonstrates that ICBN preservation no benefit to improve sensation but it is improvement of HRQOL and physical function of upper arm at 3 months after surgery.

\section{Acknowledgments}

Ms. Yada Phengsalae and Ms. Pattawia Choikrua for technical support.

\section{Footnote}

Conflicts of Interest: The authors have no conflicts of interest to declare.

Etbical Statement: The authors are accountable for all aspects of the work in ensuring that questions related to the accuracy or integrity of any part of the work are appropriately investigated and resolved. The study was 
registered on Thai Clinical Trials Registry (TCTR) number TCTR20180908002, and approved by the Office of The Committee for Research, Faculty of Medicine Ramathibodi Hospital Mahidol University (Protocol number ID 04-5908). All patients provided written informed consent before surgery.

\section{References}

1. Halsted WS. I. The Results of Operations for the Cure of Cancer of the Breast Performed at the Johns Hopkins Hospital from June, 1889, to January, 1894. Ann Surg 1894;20:497-555.

2. Fisher B, Wolmark N, Bauer M, et al. The accuracy of clinical nodal staging and of limited axillary dissection as a determinant of histologic nodal status in carcinoma of the breast. Surg Gynecol Obstet 1981;152:765-72.

3. Graversen HP, Blichert-Toft M, Andersen JA, et al. Breast cancer: risk of axillary recurrence in node-negative patients following partial dissection of the axilla. Eur J Surg Oncol 1988;14:407-12.

4. Kwan W, Jackson J, Weir LM, et al. Chronic arm morbidity after curative breast cancer treatment: prevalence and impact on quality of life. J Clin Oncol 2002;20:4242-8.

5. Assa J. The intercostobrachial nerve in radical mastectomy. J Surg Oncol 1974;6:123-6.

6. Teicher I, Poulard B, Wise L. Preservation of the intercostobrachial nerve during axillary dissection for carcinoma of the breast. Surg Gynecol Obstet 1982;155:891-2.

7. Temple WJ, Ketcham AS. Preservation of the intercostobrachial nerve during axillary dissection for breast cancer. Am J Surg 1985;150:585-8.

8. Mewa Kinoo S, Singh B. Complex Regional Pain

Cite this article as: Chirappapha $\mathrm{P}$, Arunnart M, Lertsithichai P, Supsamutchai C, Sukarayothin T, Leesombatpaiboon M. Evaluation the effect of preserving intercostobrachial nerve in axillary dissection for breast cancer patient. Gland Surg 2019;8(6):599-608. doi: 10.21037/gs.2019.10.06
Syndrome of the Breast and Chest Wall. Breast J 2016;22:366-8.

9. Abdullah TI, Iddon J, Barr L, et al. Prospective randomized controlled trial of preservation of the intercostobrachial nerve during axillary node clearance for breast cancer. Br J Surg 1998;85:1443-5.

10. Salmon RJ, Ansquer Y, Asselain B. Preservation versus section of intercostal-brachial nerve (IBN) in axillary dissection for breast cancer--a prospective randomized trial. Eur J Surg Oncol 1998;24:158-61.

11. Torresan RZ, Cabello C, Conde DM, et al. Impact of the preservation of the intercostobrachial nerve in axillary lymphadenectomy due to breast cancer. Breast J 2003;9:389-92.

12. Krittayaphong R, Bhuripanyo K, Raungratanaamporn O, et al. Reliability of Thai version of SF-36 questionnaire for the evaluation of quality of life in cardiac patients. J Med Assoc Thai 2000;83:S130-6.

13. Lim LL, Seubsman SA, Sleigh A. Thai SF-36 health survey: tests of data quality, scaling assumptions, reliability and validity in healthy men and women. Health Qual Life Outcomes 2008;6:52.

14. Kennedy CA, Beaton DE, Solway S, et al. Disabilities of the Arm, Shoulder and Hand (DASH). The DASH and QuickDASH Outcome Measure User's Manual. Third Edition. Toronto, Ontario: Institute for Work \& Health, 2011.

15. Tongprasert S, Rapipong J, Buntragulpoontawee M. The cross-cultural adaptation of the DASH questionnaire in Thai (DASH-TH). J Hand Ther 2014;27:49-54.

16. Taira N, Shimozuma K, Ohsumi S, et al. Impact of preservation of the intercostobrachial nerve during axillary dissection on sensory change and health-related quality of life 2 years after breast cancer surgery. Breast Cancer 2014;21:183-90. 